\title{
Endplate Abnormalities in Cervical Spondylotic Myelopathy and Association with Pre- and Post- operative(Single-level ACDF with a Zero-P Implant)Sagittal Parameters and Clinical Outcomes.
}

\section{Zhen Liu}

Hebei Medical University Third Affiliated Hospital

Ruo-yu Li

Hebei Medical University Third Affiliated Hospital

Zheng Wang

Hebei Medical University Third Affiliated Hospital

Da-Long Yang

Hebei Medical University Third Affiliated Hospital

Wenyuan Ding ( $\nabla$ dingwenyuan6666@sina.com )

Hebei Medical University Third Affiliated Hospital

\section{Research article}

Keywords: endplate, cervical,degeneration, disc, Zero-P

Posted Date: August 31st, 2021

DOI: https://doi.org/10.21203/rs.3.rs-841002/v1

License: (a) (i) This work is licensed under a Creative Commons Attribution 4.0 International License.

Read Full License 


\section{Abstract}

Background:Endplate abnormalities in cervical spine were closed related to pre- and post-operative symptoms, and might lead to a certain complication. However, the related researches are few, and the associations between endplate abnormalities and clinical outcomes need more evidence. Correlations between endplate abnormalities and pre- and post-operative cervical sagittal parameters also remain unknown. The purpose of this study was to explore endplate abnormalities in cervical spondylotic myelopathy, and to find potential associations of endplate abnormalities with preoperative cervical sagittal parameters, disc degeneration and symptoms, and clinical outcomes after ACDF.

Method:We enrolled 65 patients (34 males, 31 females) who were diagnosed with CSM and were subjected to single-level ACDF with a Zero-P implant at our hospital from 2015 to 2018. The patients were divided into two groups according to whether the endplate was abnormal. We conducted a retrospective study to compare the clinical and radiological results among the two groups.

Results:The average age in endplate abnormality group was higher than it in no abnormality group $(P<0.001)$. T1S was significantly higher in abnormality group than in the normal group of preoperative $(P<0.05)$ and kept significantly different from postoperative and 1 year follow up $(P<0.05)$. No significantly different was found between the two groups as for CA, CSVA, intervertebral height and preventable soft-tissue thickness. The incidence rate of interbody cage subsidence was $25.9 \%$ in 2 years follow up, which was significantly higher than that in the normal group $(P<0.05)$. The fusion rate in endplate abnormality group was $89 \%$. No significantly difference was found between the two groups. The Miyazaki grade of the disc in endplate abnormality group was significantly higher than it in the normal group $(P<0.01)$.Significant difference was detected on NDI between the two groups.

Conclusions: Endplate abnormalities in cervical spondylotic myelopathy were associated with older age, disc degeneration, decrease of intervertebral height and increase of TS before cervical surgery. Patients in endplate abnormality group showed increase in NDI pre- and post-operation, and more phenomenon of subsidence.

\section{Introduction}

Vertebral endplate abnormalities refer to the structural defects in the vertebral endplates[1]. Previous studies usually focus on the vertebral endplates in lumbar spine and several types of endplate defects were introduced, such as Schmorl's nodes, erosive defects, and arc type[2-4]. Endplate abnormalities have a close relationship with intervertebral disc degeneration (IVD) and pain in lumbar[5, 6]. Tomaszewski et al. [7] found cervical intervertebral disc degeneration had a strong correlation with endplate calcification by observation of human cadavers. And they concluded endplate calcification prevent nutrition supply to the intervertebral disc. Harada et al. [8]divided cervical endplate defects into typical abnormality and atypical abnormalities according to the morphological characteristics of endplates on MRI, and found endplate defects were associated with pain and adjacent segment 
degeneration after cervical surgery. The former is a representative of the traditional Schmorl node, and the latter (Corresponding to Schmorl's nodes, erosive defects, and arc type in the lumbar spine) is defined by greater size and shape irregularity (Fig. 1), which may eventually have a different impact on the overall degenerative spine contour and related clinical outcomes of patients.

Cervical spondylotic myelopathy (CSM) is a degenerative disorder which can lead to neurological dysfunction and neck pain. Anterior cervical discectomy and fusion (ACDF) is a standard procedure for treating patients with CSM.Previous studies demonstrated that some cervical sagittal parameters were associated with the advancing of CSM and they also influenced clinical outcomes after ACDF[9-11]. C2C7 sagittal vertical axis (CSVA), T1 slope (T1S) and C2-C7 Cobb angle (CA) are important parameters associated with cervical sagittal alignment and clinical symptoms. Increasing CA and T1S are correlated with decreasing preoperative NDI (Neck disability index), and high cSVA is an independent predictor of high preoperative NDI in patients with CSM[12]. Tang et al.[13] found C2-C7 SVA negatively correlated with SF-36 (Short Form 36-Item) and positively correlated with NDI scores, especially when cSVA $>40 \mathrm{~mm}$ postoperatively.

Some researchers noticed endplate abnormalities in the cervical spine were closed related to pre- and post-operative symptoms, and might lead to a certain complication, such as adjacent segment degeneration (ASD)[8]. However, the related researches are few, and the associations between endplate abnormalities and clinical outcomes need more evidence. Correlations between endplate abnormalities and pre- and post-operative cervical sagittal parameters also remain unknown. The purpose of this study was to explore endplate abnormalities in cervical spondylotic myelopathy, and to find potential associations of endplate abnormalities with preoperative cervical sagittal parameters, disc degeneration and symptoms, and clinical outcomes after ACDF.

\section{Materials And Methods}

We enrolled 65 patients (34 males, 31 females) who were diagnosed with CSM and were subjected to single-level ACDF with a Zero-P implant at our hospital from 2015 to 2018. Patients who underwent surgery for symptomatic degenerative lesions were included if preoperative T1 and T2 weighted MRI and X-ray images of the surgical segment could be measured and structural morphology could be evaluated.Patients who underwent other procedures during the follow-up period, those lost to follow-up, and those who did not undergo dynamic roentgen stereogrammetric analysis at least 2 years postoperatively were excluded.

The patient was given general anesthesia and supine. The right lateral incision was used to expose the operative segment.Removal of compression materials such as intervertebral disc and osteophyte, and posterior longitudinal ligament resection was performed according to the severity of dural sac compression. The endplate cartilage was scrapped in order to prepare it for bone grafting.Appropriate Zero-P was implanted into interverte-bral space. Patients were asked to move under the ground with neck support for 24 hours. 
Query the demographic information of each patient which included age, body mass index (BMI), sex, and smoking status. Other variables included operation time (minutes), length of hospitalization, operative segments, blood loss and duration of symptoms (months).

Preoperative MRI( T1-and T2-weighted )was assessed for endplate abnormalities using the method outlined by Samartzis et al.[2-4] and refer to the vertebral endplates abnormalities in lumbar spine.

NDI, SF-36 and Japanese Orthopedic Association score (JOA) were evaluated at preoperative, and postoperative, 1 year and 2 year follow-up. The phenomenon of subsidence and fusion conditions was evaluated at final follow-up. Subsidence was interpreted as a decrease of more than $3 \mathrm{~mm}$ in the height of the surgical segment between the final follow-up image and the image obtained immediately after surgery.Radiological fusion is defined as the interspinous distance motion $\leq 2^{\circ}$ on X-ray and / or the distance motion $\leq 2 \mathrm{~mm}$ on flexion-extension X-ray.The intervertebral discs were graded using the Miyazaki disc degeneration grading system[14] (grade 1-5, the higher the grade,the more serious the intervertebral disc degeneration).

\section{Sagittal parameters on the lateral radiographs were as follows:}

T1S:Draw a straight line along the superior endplate of T1 vertebral body. The angle between the line and the horizontal line is the $\mathrm{T} 1$ inclination angle.

cSVA: The distance between the plumb line passing through the center of $\mathrm{C} 2$ and the plumb line passing through the center of $\mathrm{C} 7$.

Cobb angle: Draw a straight line along the upper endplate of the upper vertebra and the lower endplate of the lower vertebra, and the angle between the two lines.

Intervertebral height: The measured height is the average of the height of the front half and the rear half.

Pre-vertebral soft-tissue thickness: It was determined by evaluating the soft tissue thickness from the front of the c3-7 vertebral body to the back of the trachea.

\section{Statistical analysis}

SPSS of Windows version 23.0 was used for statistical analysis. T test and chi square test were used to analyze the differences between the two groups. $\mathrm{P}<0.05$ was considered statistically significant.

\section{Results}

A total of 65 patients (34 males, 31 females) were included. The patients were divided into two subgroups according to the morphological characteristics at the surgical segment. $41.5 \%$ patients $(27 / 65)$ 
had endplate abnormalities. The average age in endplate abnormality group was $60.89 \pm 8.01 \mathrm{yr}$, significantly higher than the $53.76 \pm 8.52 \mathrm{yr}$ in no abnormality group $(P<0.001)$. Gender, duration of symptoms, body mass index (BMI), smoking status, operation time, length of hospitalization and blood loss were not significantly different between the two groups (Table 1).

Table 1

Comparison of the enrolled populations based on Demographic and Endplate Abnormalities

\begin{tabular}{llll} 
& $\begin{array}{l}\text { No Endplate } \\
\text { Abnormalities }(\mathrm{n}=38)\end{array}$ & $\begin{array}{l}\text { Endplate } \\
\text { Abnormalities }(\mathrm{n}=27)\end{array}$ & \\
\hline Age (year) & $53.76 \pm 8.52$ & $60.89 \pm 8.01$ & 0.001 \\
\hline Sex (male/female) & $19 / 19$ & $15 / 12$ & 0.195 \\
\hline Operation time (minutes) & $118.95 \pm 30.65$ & $114.44 \pm 22.76$ & 0.441 \\
\hline Length of hospitalization & $5.92 \pm 1.71$ & $5.89 \pm 1.93$ & 0.612 \\
\hline Operative segments & & & \\
\hline C3-4 & 12 & 9 & \\
\hline C4-5 & 11 & 11 & \\
\hline C5-6 & 13 & 6 & 0.403 \\
\hline C6-7 & 2 & 1 & 0.636 \\
\hline Blood loss & $143.16 \pm 31.63$ & $135.96 \pm 31.83$ & 0.074 \\
\hline Body mass index $(\mathrm{BMI})$ & $25.20 \pm 6.04$ & $25.91 \pm 3.83$ & 0.178 \\
\hline Current smoker $\otimes \mathrm{Y} / \mathrm{N} \otimes$ & $17 / 21$ & $13 / 14$ & $12.30 \pm 5.68$ \\
\hline Duration of symptoms & $10.21 \pm 4.22$ & & \\
\hline
\end{tabular}

Cervical sagittal parameters were compared between the two groups before and after surgery. T1S was significantly higher in abnormality group than in the normal group of preoperative $(P<0.05)$ and kept significantly different at postoperative and 1 year follow up $(P<0.05)$. T1S was not significantly different in 2 years follow up and the changes of T1S (Pre vs F/U 2y) between the two groups were not significantly different. No significantly different was found between the two groups as for CA, cSVA, intervertebral height and prevertebral soft-tissue thickness. The incidence rate of interbody cage subsidence was $25.9 \%$ in 2 years follow up, which was significantly higher than that in the normal group $(P<0.05)$. The fusion rate in endplate abnormality group was $89 \%$. No significantly difference was found between the two groups (Table 2). The Miyazaki grade of the disc in endplate abnormality group was 4.26 \pm 0.76 , which was significantly higher than the $3.66 \pm 0.91$ in the normal group $(P<0.01) \otimes T a b l e 3 \otimes$. 
Table 2

Comparisons of radiologic parameters 
No Endplate

Endplate

p-value

Abnormalities $(n=38) \quad$ Abnormalities $(n=27)$

$\operatorname{T1S}\left({ }^{\circ}\right)$

\begin{tabular}{|c|c|c|c|}
\hline Pre & $24.23 \pm 3.48$ & $27.91 \pm 5.45$ & 0.004 \\
\hline Post & $25.29 \pm 3.34$ & $28.37 \pm 5.6$ & 0.046 \\
\hline F/U 1y & $24.36 \pm 3.37$ & $27.48 \pm 5.64$ & 0.036 \\
\hline F/U 2y & $25.17 \pm 3.84$ & $27.68 \pm 5.56$ & 0.068 \\
\hline$\triangle \mathrm{T} 1 \mathrm{~s}\left({ }^{\circ}\right)($ Pre vs $\mathrm{F} / \mathrm{U} 2 \mathrm{y})$ & $0.94 \pm 2.47$ & $-0.23 \pm 3.16$ & 0.100 \\
\hline \multicolumn{4}{|l|}{$\mathrm{CA}\left({ }^{\circ}\right)$} \\
\hline Preoperative & $12.93 \pm 5.94$ & $13.82 \pm 2.07$ & 0.052 \\
\hline Post & $15.74 \pm 4.58$ & $16.86 \pm 2.07$ & 0.053 \\
\hline F/U 1y & $15.53 \pm 4.83$ & $16 \pm 1.75$ & 0.177 \\
\hline$F / U 2 y$ & $14.52 \pm 4.72$ & $14.71 \pm 1.55$ & 0.103 \\
\hline$\triangle \mathrm{CA}\left({ }^{\circ}\right)($ Pre vs F/U 2y) & $1.58 \pm 3.05$ & $0.89 \pm 1.77$ & 0.170 \\
\hline \multicolumn{4}{|l|}{ cSVA (mm) } \\
\hline Pre & $18.86 \pm 4.78$ & $17.07 \pm 3.12$ & 0.073 \\
\hline Post & $23.4 \pm 4.53$ & $22.18 \pm 2.32$ & 0.401 \\
\hline F/U 1y & $21.92 \pm 4.59$ & $21.07 \pm 2.01$ & 0.567 \\
\hline$F / U 2 y$ & $22.46 \pm 4.50$ & $21.12 \pm 2.15$ & 0.315 \\
\hline$\triangle \mathrm{cSVA}(\mathrm{mm})($ Pre vs F/U 2y) & $3.60 \pm 2.41$ & $4.05 \pm 2.71$ & 0.490 \\
\hline \multicolumn{4}{|l|}{ Intervertebral height (mm) } \\
\hline Pre & $5.36 \pm 1.41$ & $4.26 \pm 1.46$ & 0.008 \\
\hline Post & $7.67 \pm 1.35$ & $7.97 \pm 0.90$ & 0.288 \\
\hline F/U 1y & $6.79 \pm 1.46$ & $7.00 \pm 0.73$ & 0.775 \\
\hline$F / U 2 y$ & $6.46 \pm 1.37$ & $5.82 \pm 0.67$ & 0.027 \\
\hline$\triangle \mathrm{IH}($ Pre vs $F / U 2 \mathrm{y})$ & $1.10 \pm 0.99$ & $1.56 \pm 1.77$ & 0.220 \\
\hline
\end{tabular}

Prevertebral soft-tissue

Thickness (mm)

$\begin{array}{llrl}\text { Pre } & 12.72 \pm 3.05 & 14.11 \pm 3.16 & 0.080 \\ \text { Post } & 16.83 \pm 2.91 & 17.46 \pm 3.28 & 0.460\end{array}$




\begin{tabular}{llll}
\hline F/U 1y & $14.03 \pm 2.79$ & $14.26 \pm 2.76$ & 0.747 \\
\hline F/U 2y & $13.24 \pm 2.39$ & $13.55 \pm 2.60$ & 0.616 \\
\hline$\triangle($ Pre vs F/U 2y) & $0.52 \pm 1.70$ & $-0.22 \pm 1.37$ & 0.074 \\
\hline Subsidence & $5.3 \%$ & $25.9 \%$ & 0.044 \\
\hline Fusion & $95 \%$ & $89 \%$ & 0.642
\end{tabular}

Table 3

Comparison of Miyazaki grading of cervical disc degeneration in two groups

\begin{tabular}{llll} 
& No Endplate & Endplate & p-value \\
& Abnormalities $(n=38)$ & Abnormalities $(n=27)$ & \\
\hline Miyazaki grading & $3.66 \pm 0.91$ & $4.26 \pm 0.76$ & 0.006
\end{tabular}

Cervical endplate abnormalities had associations with symptoms and clinical outcomes before and after ACDF. SF-36 and JOA scores were increasing and NDI was decreasing after the surgery both in the normal group and endplate abnormality group. Significant difference was detected on NDI between the two groups. Abnormality group had significantly higher preoperative NDI scores and the scores remained higher in postoperative and 1 year follow up compared to the normal group $(P<0.05)$. There was no significant difference in pre- and post-operative JOA and SF-36 between the two groups (Table 4).

Table 4

Quality of Life Parameters 
No Endplate $\quad$ Endplate p-value

Abnormalities $(n=38)) \quad$ Abnormalities $(n=27)$

JOA

\begin{tabular}{|c|c|c|c|}
\hline Pre & $10.58 \pm 1.52$ & $10.15 \pm 1.88$ & 0.215 \\
\hline Post & $13.13 \pm 1.51$ & $13.11 \pm 0.97$ & 0.885 \\
\hline F/U 1y & $12.90 \pm 1.83$ & $13.22 \pm 1.60$ & 0.431 \\
\hline F/U 2y & $13.26 \pm 1.81$ & $13.52 \pm 1.74$ & 0.512 \\
\hline$\triangle \mathrm{JOA}($ Pre vs Post) & $2.55 \pm 1.52$ & $2.96 \pm 1.79$ & 0.101 \\
\hline$\triangle \mathrm{JOA}($ Pre $v s \mathrm{~F} / \mathrm{U} 2 \mathrm{y})$ & $2.68 \pm 1.89$ & $3.37 \pm 2.13$ & 0.075 \\
\hline \multicolumn{4}{|l|}{ NDI } \\
\hline Pre & $19.53 \pm 4.94$ & $23.00 \pm 4.18$ & 0.012 \\
\hline Post & $14.74 \pm 4.06$ & $17.63 \pm 4.78$ & 0.011 \\
\hline F/U 1y & $12.97 \pm 3.35$ & $15.78 \pm 4.37$ & 0.020 \\
\hline F/U 2y & $12.08 \pm 3.59$ & $13.56 \pm 2.82$ & 0.080 \\
\hline$\triangle \mathrm{NDI}$ (Pre vs Post ) & $-4.79 \pm 2.94$ & $-5.37 \pm 3.21$ & 0.678 \\
\hline$\triangle \mathrm{NDI}(\operatorname{Pre} v s \mathrm{~F} / \mathrm{U} 2 \mathrm{y})$ & $-7.45 \pm 3.02$ & $-9.44 \pm 5.17$ & 0.145 \\
\hline \multicolumn{4}{|l|}{ SF-36 } \\
\hline Pre & $43.90 \pm 6.52$ & $41.70 \pm 5.91$ & 0.150 \\
\hline Post & $52.32 \pm 7.38$ & $51.89 \pm 6.80$ & 0.813 \\
\hline F/U 1y & $53.18 \pm 7.06$ & $52.96 \pm 6.05$ & 0.895 \\
\hline $\mathrm{F} / \mathrm{U} 2 \mathrm{y}$ & $53.74 \pm 7.92$ & $53.33 \pm 5.38$ & 0.808 \\
\hline$\triangle$ SF-36(Pre vs Post) & $8.42 \pm 4.66$ & $10.19 \pm 5.47$ & 0.408 \\
\hline$\triangle$ SF-36(Pre vs F/U 2y) & $9.84 \pm 4.18$ & $11.63 \pm 5.74$ & 0.184 \\
\hline
\end{tabular}

\section{Discussion}

The endplate is composed of bone endplate and cartilage endplate. As an important part of the vertebral structure, endplate plays a role of mechanical conduction. At the same time, endplate is one of the main ways for nutrients to reach intervertebral disc[15]. Previous studies found endplate abnormalities were common in lumbar spine[16]. The morphologies of endplate abnormalities observed on MRI included Schmorl's nodes, notch type, erosive defects, rim defects and et al[ $[2,4]$. Endplate abnormalities had close associations with intervertebral disc degeneration, Modic changes and clinical symptoms $[5,6,17]$. Age, 
slippage and endplate morphology are potential risk factors for endplate abnormalities. However, little is known about the relationship between endplate abnormalities and cervical sagittal parameters before and after the cervical surgery. And whether the endplate abnormalities have influence on symptoms and clinical outcomes in cervical need more evidence.

This study compared age, gender, $\mathrm{BMI}$, smoking status and duration of symptoms between the normal group and endplate abnormality group, and explored whether operation time, length of hospitalization and blood loss were significantly different between the two groups (Table 1). Only age was found significantly different between them, which was consistent with previous research[3]. This illustrated that endplate abnormality may not influence the procedure of the cervical surgery.

Previous studies demonstrated that some cervical sagittal parameters were associated with endplate signal changes (Modic changes) and clinical outcomes. T1S was defined as the angle between a horizontal line and the superior endplate of T1[18]. It was found that T1S was significantly higher in patients with Modic changes (MCs) than normal group, and disc in the MCs group also had more severe disc degeneration[19]. In this study, we found preoperative T1S was significantly higher in abnormality group than in the normal group $(P<0.05)$, and intervertebral height at the surgery segment was significantly lower in abnormality group $(\mathrm{P}<0.05)$ (Table 2$)$. Intervertebral disc degeneration was significantly more severe in abnormality group (Table 3 ). Considering changes of endplate morphology impair its permeability and associated with disc degeneration, the endplate plays an important role in the development of disc degeneration[20]. Lv B and colleagues believed cervical balance impaired when T1S is greater than $25^{\circ}$, which may accelerate disc degeneration and MCs at C5-6[11] . T1S kept significantly different in postoperative and 1 year follow up $(P<0.05)$ (Table 2). To sum up, the changes of T1S are caused by three factors: first, intervertebral disc degeneration leads to the loss of intervertebral height, which leads to the increase of T1S; second, some endplate abnormalities lead to vertebral collapse, which eventually leads to the increase of T1S; third, patients with endplate abnormalities have more severe symptoms, limited cervical movement, and forced posture, which leads to the increase of T1S.This study didn't find prevertebral soft-tissue thickness. Cobb angles and cSVA were associated with endplate abnormalities. NDI decreased, JOA and SF-36 increased in both groups after surgery, which means the patients can achieve good clinical outcomes by the surgery. NDI was significantly higher in endplate abnormality group before the surgery and kept significantly different in postoperative and 1 year follow up $(P<0.05)$ (Table 4). This trend was similar to T1S.Song and yang[21]pointed out that the Schmorl node can create a channel for disc herniation in patients with Scheuermann's disease,which leads to rapid onset of spinal cord injury. Although rare,this example suggests that structural endplate abnormalities may increase the risk of cervical spondylosis. Similar principles can be applied to this study, and can explain why endplate abnormalities lead to a higher incidence of symptoms of cervical spondylotic myelopathy.

Subsidence indicated loss of surgical segment height after the surgery. Intervertebral height in endplate abnormality group was significantly lower in 2 years follow up compared to the normal group $(P<0.05)$ (Table 2). The main reason for the low fusion rate in the abnormal endplate group is that the endplate 
destroys the vertebral bone and causes the fusion cage to sink. On the other hand, it is considered that some endplates are not treated or not completely treated during the operation, which causes the fusion cage to sink. The decrease of intervertebral height (Pre vs F/U 2y) showed no significant difference between the two groups. Intervertebral height changes are affected by many factors, intervertebral disc degeneration will lead to the loss of intervertebral height, settlement will lead to the loss of intervertebral height, under the comprehensive influence of the above results are not statistically significant.ACDF surgery using Zero-P implants showed high fusion rates. In this study, both groups had satisfied fusion rates[22]. No significant difference was found between them (Table 2).

\section{Limitations}

This retrospective study includes some limitations. First, endplate abnormalities had sub-types according to their morphological characteristics, we only divided the patients into two groups and didn't analyse different endplate abnormalities separately because of small sample size. In addition, the relationship between endplate abnormalities and disc degeneration, cervical sagittal parameters and clinical outcomes needs prospective study to confirm.

\section{Conclusion}

Endplate abnormalities in cervical spondylotic myelopathy were associated with older age, disc degeneration, decrease of intervertebral height and increase of T1S before cervical surgery. Patients in endplate abnormality group showed increase of NDI pre- and post operation, and more phenomenon of subsidence. Nevertheless, the author believes that ACDF should be performed in CSM patients with surgical indications, regardless of whether the endplate is abnormal or not. Whether the endplate is abnormal is only a better judgment of the postoperative effect.

\section{Abbreviations}

IVD:Intervertebral disc degeneration;CSM:Cervical spondylotic myelopathy; ACDF:Anterior cervical discectomy and fusion;CSVA:C2-C7 sagittal vertical axis;T1S:T1 slope;CA:C2-C7 Cobb angle;NDI: Neck disability index;SF-36:Short Form 36-Item ;ASD:Adjacent segment degeneration;BMI:Body mass index;JOA:Japanese Orthopedic Association score ;MCs:Modic changes .

\section{Declarations}

\section{Ethics approval and consent to participate}

This article does not contain any studies with human participants or animals performed by any of the authors. 


\section{Consent for publication}

Written informed consent for publication was obtained from all participants.

\section{Availability of data and materials}

The datasets generated and analyzed during the current study are available from the corresponding author on reasonable request.

\section{Competing interests}

The authors report no confflicts of interest in this work.

\section{Funding}

None.

\section{Authors' contributions}

WYD conceived and designed the study; ZL and ZW collected; ZL, RYL, and ZW analyzed and interpreted the patient data. $Z \mathrm{~L}$ and RYL wrote the paper. All authors read and approved the final manuscript. All authors have read the journal policies and have no issues relating to journal policies. All authors have seen the manuscript and approved to submit to your journal. The work described has not been submitted elsewhere for publication, in whole or in part. The authors read and approved the final manuscript.

\section{Acknowledgements}

At the point of finishing this paper, l'd like to express my sincere thanks to all those who have lent me hands in the course of my writing this paper. Without their help, it would be much harder for me to finish my study and this paper.

\section{References}

1. Lawan A, Leung A, Battie MC. Vertebral endplate defects: nomenclature, classification and measurement methods: a scoping review. Eur Spine J. 2020;29(6):1397-409.

2. Brayda-Bruno $M$, et al. Endplate lesions in the lumbar spine: a novel MRI-based classification scheme and epidemiology in low back pain patients. Eur Spine J. 2018;27(11):2854-61. 
3. Wang Y, Videman T, Battie MC. Lumbar vertebral endplate lesions: prevalence, classification, and association with age. Spine (Phila Pa 1976). 2012;37(17):1432-9.

4. Li R, et al. Lumbar Vertebral Endplate Defects on Magnetic Resonance Imaging in Degenerative Spondylolisthesis: Novel Classification, Characteristics, and Correlative Factor Analysis. World Neurosurg. 2020;141:e423-30.

5. Luoma K, et al. Chronic low back pain in relation to Modic changes, bony endplate lesions, and disc degeneration in a prospective MRI study. Eur Spine J. 2016;25(9):2873-81.

6. Munir S, et al. Endplate Defect Is Heritable, Associated With Low Back Pain and Triggers Intervertebral Disc Degeneration: A Longitudinal Study From TwinsUK. Spine (Phila Pa 1976). 2018;43(21):1496-501.

7. Tomaszewski KA, et al. Endplate calcification and cervical intervertebral disc degeneration: the role of endplate marrow contact channel occlusion. Folia Morphol (Warsz). 2015;74(1):84-92.

8. Harada GK, et al. Cervical Spine Endplate Abnormalities and Association With Pain, Disability, and Adjacent Segment Degeneration After Anterior Cervical Discectomy and Fusion. Spine (Phila Pa 1976). 2020;45(15):E917-26.

9. Fan XW, et al. The change of cervical sagittal parameters plays an important role in clinical outcomes of cervical spondylotic myelopathy after multi-level anterior cervical discectomy and fusion. J Orthop Surg Res. 2019;14(1):429.

10. Nicholson KJ, et al. Cervical Sagittal Range of Motion as a Predictor of Symptom Severity in Cervical Spondylotic Myelopathy. Spine (Phila Pa 1976). 2018;43(13):883-9.

11. Lv B, et al. C7 slope and its association with serum lipid levels and Modic changes in patients with cervical spondylotic myelopathy. J Pain Res. 2019;12:1767-76.

12. Iyer S, et al. Impact of Cervical Sagittal Alignment Parameters on Neck Disability. Spine (Phila Pa 1976). 2016;41(5):371-7.

13. Tang JA, et al., The impact of standing regional cervical sagittal alignment on outcomes in posterior cervical fusion surgery. Neurosurgery, 2015. 76 Suppl 1: p. S14-21; discussion S21.

14. Miyazaki $\mathrm{M}$, et al. Kinematic analysis of the relationship between the grade of disc degeneration and motion unit of the cervical spine. Spine (Phila Pa 1976). 2008;33(2):187-93.

15. Wu Y, et al. Region and strain-dependent diffusivities of glucose and lactate in healthy human cartilage endplate. J Biomech. 2016;49(13):2756-62.

16. Wang Y, Videman T, Battie MC. Morphometrics and lesions of vertebral end plates are associated with lumbar disc degeneration: evidence from cadaveric spines. J Bone Joint Surg Am. 2013;95(5):e26.

17. Feng Z, et al. Lumbar Vertebral Endplate Defects on Magnetic Resonance Images: Classification, Distribution Patterns, and Associations with Modic Changes and Disc Degeneration. Spine (Phila Pa 1976). 2018;43(13):919-27. 
18. Ling FP, et al. Which parameters are relevant in sagittal balance analysis of the cervical spine? A literature review. Eur Spine J. 2018;27(Suppl 1):8-15.

19. Li J, et al. Modic changes of the cervical spine: T1 slope and its impact on axial neck pain. J Pain Res. 2017;10:2041-5.

20. Fields AJ, et al. Contribution of the endplates to disc degeneration. Curr Mol Biol Rep. 2018;4(4):15160.

21. Song KS, Yang JJ. Acutely progressing paraplegia caused by traumatic disc herniation through posterior Schmorl's node opening into the spinal canal in lumbar Scheuermann's disease. Spine (Phila Pa 1976). 2011;36(24):E1588-91.

22. Yin $\mathrm{M}$, et al. The new Zero-P implant can effectively reduce the risk of postoperative dysphagia and complications compared with the traditional anterior cage and plate: a systematic review and metaanalysis. BMC Musculoskelet Disord. 2016;17(1):430.

\section{Figures}

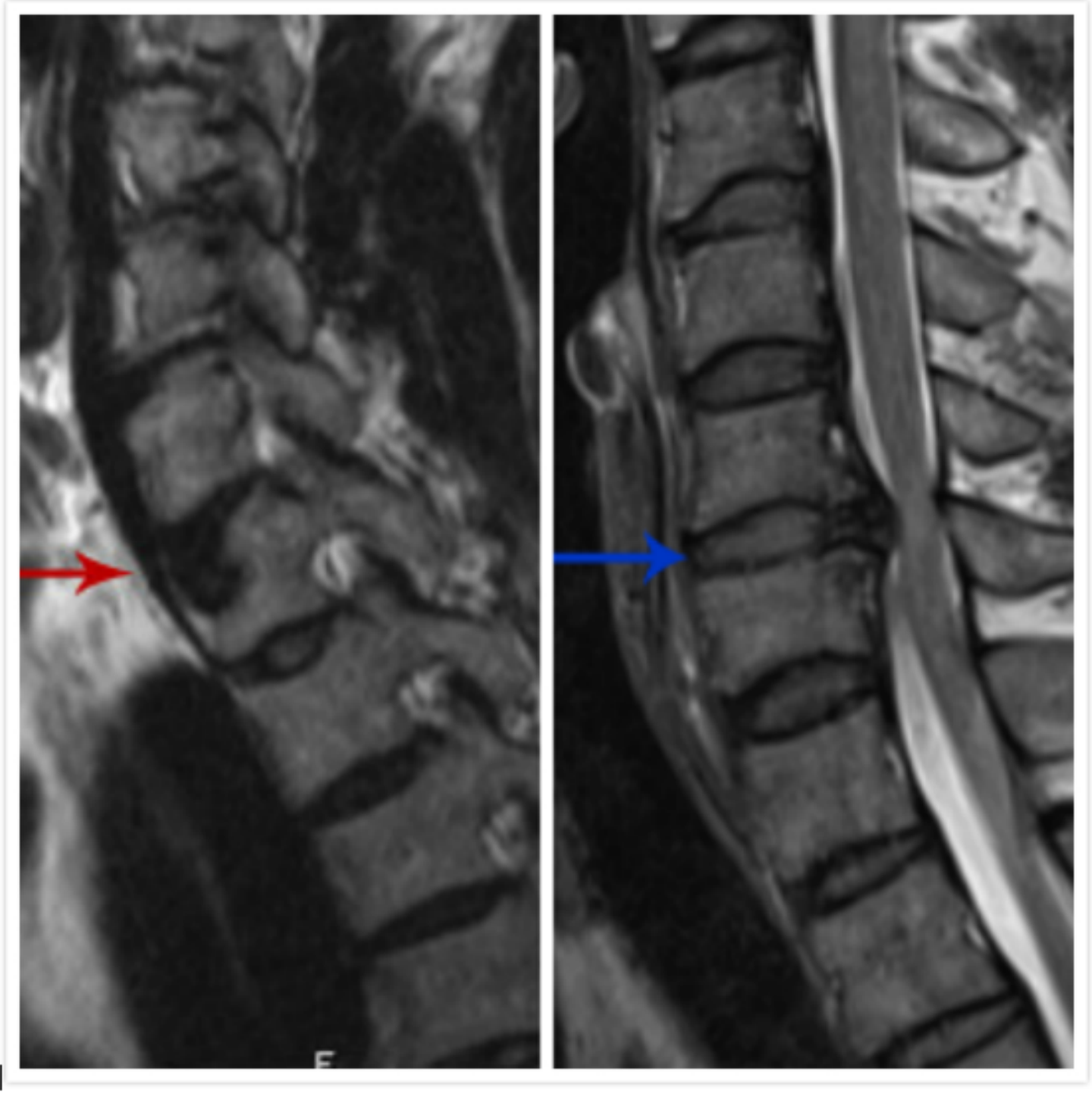




\section{Figure 1}

Typical (left) and atypical (right) endplate abnormal sample images. The typical endplate abnormality (red arrow) refers to the traditional Schmorl's node. Atypical endplate abnormalities (blue arrows) are irregular in shape and size. It is not discussed separately in this paper. 